\title{
Discovery of Diosgenin as Potent and Selective Inhibitors Against Human Carboxylesterase 2
}

\section{Xiao-Dong Li ${ }^{1,2, a}$, Hong-Hong $\mathrm{Ma}^{2, a}$, Jun-Qing Gao ${ }^{1}$, Yi-Shu Zhao ${ }^{2}$, Xing-Kai Qian², Guang-Bo Ge ${ }^{2}$, Li-Wei Zou ${ }^{2, a *}$, Zong-Jun Liu ${ }^{1, a *}$ and Ling Yang ${ }^{2}$}

${ }^{1}$ Department of Cardiology, Putuo Hospital, Shanghai University of Traditional Chinese Medicine, Shanghai, China

${ }^{2}$ Institute of Interdisciplinary Integrative Medicine Research, Shanghai University

of Traditional Chinese Medicine, Shanghai, China

${ }^{a}$ These Authors have Contributed Equally to this Work

*Corresponding Author: Li-Wei Zou, Institute of Interdisciplinary Integrative Medicine Research, Shanghai University of Traditional Chinese Medicine, Shanghai, China and Zong-Jun Liu, Department of Cardiology, Putuo Hospital, Shanghai University of Traditional Chinese Medicine, Shanghai, China.
Received: November 02, 2021

Published: November 18, 2021

(C) All rights are reserved by Xiao-Dong Li., et al.

\begin{abstract}
Paris polyphylla,a famous traditional Chinese herb, has the effects of clearing away heat and toxin, relieving swelling and pain, cooling the liver, and calming convulsion. Human carboxylesterase 2 (hCES2A), one of the most principal drug-metabolizing enzymes, catalyzes the hydrolysis of a variety of endogenous esters and environmental toxicants. In this study, the inhibitory effects against hCES2A of four active ingredients of Paris Polyphylla were assayed using diacetylfluorescein (FD) as a specific optical substrate for hCES2A. Among the four compounds, diosgenin displayed significantly inhibitory activity against hCES2A. Further investigations demonstrate that diosgenin exhibits strong hCES2A inhibition selectivity over other human serine hydrolases, including hCES1A, dipeptidyl peptidase IV (DPP-IV), butyrylcholinesterase (BChE), pancreatic lipase (PL), and thrombin. According to its inhibition kinetic result, diosgenin was a mixed model type inhibitor, and $K_{\mathrm{i}}$ was $1.15 \mu \mathrm{M}$. Molecular docking showed that diosgenin could dock well to the active site and $\mathrm{Z}$ site of hCES2A, and the hydrophobic interactions might be the major ways for the binding of diosgenin on hCES2A. All results indicated that diosgenin is a potent and selective hCES2A inhibitor, which may potentially affect the metabolism of endogenous substances or cause unpredictable DDI by inhibiting the activity of hCES2A.
\end{abstract}
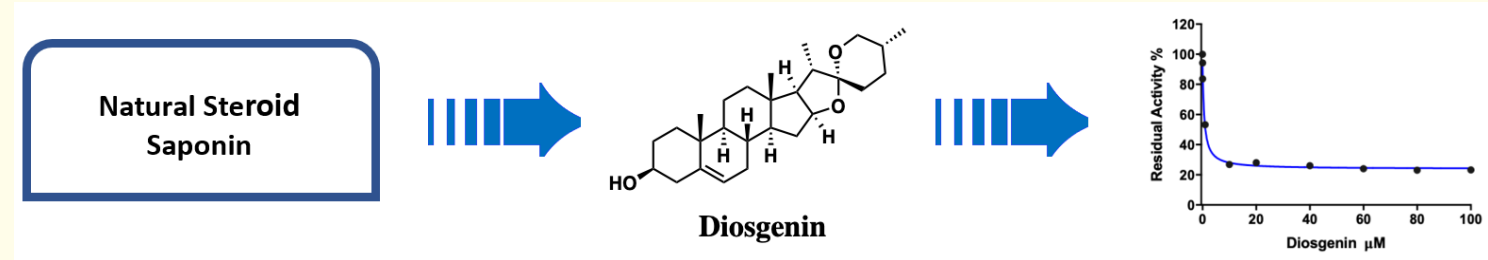

Keywords: Paris Polyphylla; Diosgenin; Human Carboxylesterase 2; Inhibitor; Molecular Docking Simulations

\section{Introduction}

Paris polyphylla, the main medicinal parts are rhizomes of Parispolyphylla Smith var. yunnanensis (Franch.) Hand. -Mazz, has been used as a famous ingredient with the same origin as medicine and food in Chinese traditional medicines for thousands of years. In recent decades, Paris polyphylla was often be used as anticancer 
medicine such as breast cancer [10], and lung cancer [3]. Polyphyllin I, VI, VII and diosgenin as a naturally occurring steroidal sapogenin has been shown to be hypocholesterolemic, anti-inflammatory and anti-cancer, especially in terms of anti-cancer, diosgenin has been reported to have pro-apoptotic and anti-cancer properties for various cancers in vitro and in vivo [19]. Diosgenin has abundant pharmacological activity, there are no reports of a related effect between diosgenin and other important drug metabolism enzymes in the human body, for example, Carboxylesterases (CEs), as an important member of the serine hydrolase superfamily. CEs is widely distributed in various organs of human body, such as liver, intestine, lung, kidney, etc. In human, hCES1A and hCES2A are two major carboxylesterase isoforms involved in endogenous and xenobiotic metabolism, which have been extensively investigated over the past decades [29,32]. Studies have shown that the former is mainly expressed in the liver and tends to hydrolyze ester or amide substrates containing large acyl or small alcohol groups $[2,6]$; while the latter, which are expressed mainly in the intestinal epithelium, with the highest content in the duodenum, and tends to hydrolyze relatively small acyl or large alcohol group [17,20,33].

As the main carboxylesterase in human intestinal tract and tumor tissue, the activity of hCES2A can significantly affect the bioavailability of oral prodrugs and the efficacy of ester anticancer drugs [18]. For example, hCES2A can hydrolyze irinotecan into active metabolite SN38 causing a series of adverse reactions, such as delayed diarrhea, which affect the efficacy and increase toxicity [21]. When the activity of hCES2A was inhibited, the probability of such adverse reactions will be greatly reduced. Therefore, the discovery and search of efficient hCES2A inhibition is imperative, so far, researchers have discovered a variety of hCES2A inhibitors from natural products and traditional Chinese medicines, such as flavonoids [11,12], tanshinones [5], triterpenoids [38], and alkaloids [37], which have strong inhibitory effects on hCES2A $[11,23,27]$. In addition, we are delighted to find Polyphyllin I affect the expression of E-cadherin and N-cadherin, which related to the regulation of EMT (epithelial-mesenchymal transition), and finally inhibiting the cell migration and invasion of human colon cancer HCT116 cells and SW620 cells [34]. Finding a natural product that can selectively inhibit hCES2A in Paris polyphylla can achieve the effect of combining Paris polyphylla and irinotecan to reduce toxicity. In the current experiment, fluorescein diacetate (FD) was used as a fluorescent probe substrate to study the inhibitory effect of four active ingredients of Paris polyphylla on hCES2A, and enzyme kinetics was also detected. At the same time, the inhibitory effects of diosgenin on other serine hydrolases were compared. The interaction mechanism between diosgenin and hCES2A was investigated by molecular docking.

\section{Materials and Methods}

Chemicals and reagents

Diosgenin and Paris polyphylla Smith var. yunnanensis (Franch.) Hand.-Mazz. saponin were obtained from Bide Pharmaceutical Co., Ltd. (Shanghai, China). Polyphyllin I, VI, VII were purchased from MeilunBio Co., Ltd. (Dalian, China). The ${ }^{1} \mathrm{H}$ NMR and ${ }^{13} \mathrm{C}$ NMR spectra of these compounds were recorded using an NMR spectrometer. Human liver microsomes (HLM from 50 donors, lot X008067) were obtained from Bioreclamation IVT (Baltimore, MD, USA). Human plasma was purchased from Research Institute for Liver Diseases (Shanghai) Ltd Co (RILD). Thrombin (isolated from human plasma) was purchased from HYPHEN BioMed (France, Lot F1700752P2). The hCES2A substrate fluorescent diacetate (FD) was purchased from TCI (Tokyo, Japan). The substrate of hCES1A, D-luciferin methyl ester (DME) and dipeptidyl peptidase IV (DPP-IV) substrate GP-BAN was synthesized in our laboratory. Fluorescein detection reagent (LDR) was purchased from Promega Biotech (Madison, USA). 4-Methylumbelliferyl oleate (4-MUO), a lipase fluorescent substrate, was purchased from Sigma-Aldrich Co., Ltd. Fluorescent substrates for bovine serum albumin (BSA), Z-GlyGly-Arg-AMC acetate and human thrombin were purchased from Medchem Express (Shanghai, China). 5,5'-dithiobis(2-nitrobenzoic acid) (DTNB) and Butyrylcholinesterase (BChE) were purchased from Sigma-Aldrich. All other reagents were HPLC grade or the highest grade commercially available.

\section{Enzyme inhibition assays}

\section{hCES2A inhibition assay}

The inhibitory effect on hCES2A was studied using FD as a specific probe substrate. First, premix $194 \mu \mathrm{L}$ PBS (Phosphate buffered saline, $0.1 \mathrm{mM}$, pH 7.4) with $2 \mu \mathrm{L}$ inhibitor/DMSO at serial concentrations $(0 \sim 100 \mu \mathrm{M})$ and $2 \mu \mathrm{L}$ HLM (final concentration: $2 \mu \mathrm{g} / \mathrm{mL}$ ) to $196 \mu \mathrm{L}$ HLM (Human Liver Microsome) system. Subsequently, add the black standard 96-well plate, vortex to mix well, and preincubate at $37^{\circ} \mathrm{C}$ for $3 \mathrm{~min}$. Finally, add FD to start the reaction and 
perform fluorescence analysis. The hydrolyzed metabolites of FD were analyzed in real time using a fluorescence microplate reader (SpectraMax® iD3) using excitation and emission wavelengths of $480 \mathrm{~nm}$ and $525 \mathrm{~nm}$ (gain = 500), respectively.

\section{hCES1A inhibition assay}

Slightly different from the hCES2A inhibition experiment, the hCES1A incubation system includes DMSO/inhibitor, human liver microsomes $(1 \mu \mathrm{g} / \mathrm{mL})$, substrate DME (3 $\mu \mathrm{M})$ and PBS. In brief, combine different concentrations of DMSO/inhibitor with PBS. After pre-incubation $3 \mathrm{~min}$, then add DME to the hCES1A incubation system to start the reaction and incubate the hCES1A system at $37^{\circ} \mathrm{C}[29]$.

\section{Pancreatic lipase (PL) inhibition assay}

Similar to the hCES2A inhibition test, the PL incubation system includes PBS (Citric acid--disodium hydrogenphosphatete) and DMSO/Inhibitor on a black standard 96-well plate, subsequently, incubating the system for $10 \mathrm{~min}$, at $37^{\circ} \mathrm{C}$. After incubating, add substrate 4-MUO (4-methylumbelliferone, $3 \mu \mathrm{M}$ ) to the system to start the reaction and then placed in a fully automated fluorescent microplate reader for fluorescence analysis, using excitation and emission wavelengths of $340 \mathrm{~nm}$ and $460 \mathrm{~nm}$, respectively (Gain $=500$ ) [7].

\section{DPP-IV inhibition assay}

The DPP-IV incubation system, which contains DMSO/inhibitor, human plasma, substrate GP-BAN $(100 \mu \mathrm{M})$ and PBS [34]. The system (without substrate) was shaken on a scroll and incubated in a metal bath at $37^{\circ} \mathrm{C}$ for $3 \mathrm{~min}$. After incubation, the substrate GPBAN was added to initiate the reaction. Then, incubate at $37^{\circ} \mathrm{C}$ for $20 \mathrm{~min}$, add the equal volume ice-acetonitrile and shake it violently for $0.5-1$ min to stop the reaction, put the sample on ice and cool it until centrifugation for $10 \mathrm{~min}$, and finally absorb 100-200 $\mu \mathrm{L}$ supernatant of the centrifuge solution for fluorescence analysis on the enzyme plate analyzer. Excitement and emission wavelengths (gain $=500$ ) at $430 \mathrm{~nm}$ and $535 \mathrm{~nm}$ were used, respectively. At the same time, three control groups were set. One group without inhibitor (adding constant volume of DMSO) was used as 100\% enzyme activity control, the second group without substrate (adding constant volume of Buffer) was used to measure background fluorescence, and the third group was positive inhibitor (sitagliptin) [40].
Thrombin inhibition assay

Similarly, the thrombin microsome incubation system contains inhibitor/DMSO, Tris- $\mathrm{HCl}$, thrombin $(0.1 \mathrm{NIH} / \mathrm{mL})$, substrate ZGly-Gly-Arg-AMC (5 $\mu \mathrm{M}), 10 \mu \mathrm{L}$ BSA $(0.1 \mathrm{mg} / \mathrm{mL})$.

\section{BChE inhibition assay}

The BChEincubationsystem contents developer 5-5'-dithiobis(2nitrobenzoic acid) (DTNB) (1000 $\mu \mathrm{M})$, PBS (0.1M), human serum, and DMSO/inhibitor. At the same time set three parallel, set three control groups. They were divided into non-inhibitor group (control of $100 \%$ enzyme activity and constant volume of DMSO), butyryl cholinesterase group (constant volume of PBS for background fluorescence determination), and positive inhibitor group (galantamine hydrobromide, $10 \mathrm{M}$ ). The BTch in this experiment was dissolved in Milliore water, the system was incubated for $3 \mathrm{~min}$, then $10 \mu \mathrm{L}$ BTch was added, and the absorbance (ABS) of the product was determined by automatic luciferase plate analyzer. The ABS of the product was measured at the wavelength of $412 \mathrm{~nm}$, the time interval of 60 seconds and the detection time of 20 minutes. The final concentration of DMSO was $1 \%$. After the test is complete, calculate the slope of the test results. The residual activity of butyryl cholinesterase was calculated by the following equation, residual activity $(\%)=($ slope in the presence of inhibitor $) /$ slope of negative control (DMSO only) ×100\% [37].

Inhibition kinetic analysis

We defined $\mathrm{IC}_{50}$ as the concentration of inhibitor when the enzyme activity decreased by $50 \%$. Under the same culture conditions, we measured the $\mathrm{IC}_{50}$ in the presence of the inhibitor. $\mathrm{A}$ series of concentrations of diosgenin solution and different concentrations of FD were used to measure the inhibition constant $\left(K_{\mathrm{i}}\right)$ value of diosgenin to hCES2A in HLM. In order to determine the type (competitive inhibition, non-competitive inhibition and mixed inhibition) of inhibition kinetics of diosgenin, various concentrations of FD and different inhibitor concentrations were utilized to determine the corresponding reaction rates. The inhibition kinetic type was evaluated by determining the intersection point in the Lineweaver-Burk plots.

\section{Statistical analysis}

All the experimental data were performed in 3 sets of parallel experiments, and the results were expressed as the mean \pm SD. The 
$\mathrm{IC}_{50}$ value was analyzed by the nonlinear regression using GraphPad Prism 8.0 software (GraphPad Software, Inc., La Jolla, USA).

\section{Molecular docking}

AutoDock Vina (Version1.1.2) was used for molecular docking simulation, further exploring the interactions between ligand and enzyme. We use the previously reported homology modeling hCES2A structure for molecular docking simulation (Trott and Olson; Huo., et al.). The structure of hCES2A was processed as follows. We are removing water molecules, adding the hydrogens, merging non-polar hydrogens, and using the Kollman method for adding charges. Subsequently, bond lengths and bond angles of diosgenin were optimized with energy minimization, and the grid box was set to $80 \times 80 \times 80 \AA^{3}$ with a spacing of $0.375 \AA$ to wrap the active pocket. Finally, the binding poses with the lowest energy were analyzed, and the interaction analysis and visualization were conducted by Discovery Studio Visualizer (BIOVIA Discovery Studio 2019, Dassault Systèmes, SanDiego, USA) and PyMOL (The PyMOL Molecular Graphics System Version 2.3, Schrödinger, LLC., New York City, USA).

\section{Cytotoxicity test (Caco-2) of diosgenin}

The CCK8 assay evaluated the cytotoxicity induced by diosgenin and polyphyllins. Cells ( $0.5 \times 10^{4}$ cells/well) were seeded onto 96 well microtiter plates and cultured in a humidified atmosphere of $95 \%$ air and $5 \% \mathrm{CO} 2$ at a constant temperature of $37^{\circ} \mathrm{C}$. Then the cultured cells were treated at various concentrations of tested compounds for $48 \mathrm{~h}$. The final concentration of DMSO or absolute ethanol used in all experiments was below $2 \%(\mathrm{~V} / \mathrm{V})$. Following the treatments, cells were exposed to CCK8 for $1 \mathrm{~h}$ at $37^{\circ} \mathrm{C}$. Then, absorbance was measured at $450 \mathrm{~nm}$. The viability of cells was expressed as a percentage of the absorbance measured in control cells.

\section{Results and Discussion}

\section{Inhibitory effects of Diosgenin towards hCES2A}

The inhibitory effects of polyphyllin I, VI, VII, and diosgenin towards hCES2A were investigated firstly. FD hydrolysis was used to screen the residual activities of hCES2A in the human liver microsomes (HLMs). Preliminary screening of these compounds inhibitory effect on hCES2A using different inhibitor concentrations showed that diosgenin displayed a better inhibitory effect towards hCES2A over polyphyllin I, VI, VII. Further studies on the inhibitory effects of these compounds against hCES2A were assayed. As shown in table 1, Polyphyllin I displayed poor inhibitory effects on hCES2A, while polyphyllin VI and polyphyllin VII displayed strong inhibitory effects on hCES2A with lower $\mathrm{IC}_{50}$ value about $10 \mu \mathrm{M}$. In addition, the diosgenin had obvious inhibitory effect towards hCES2A. The $\mathrm{IC}_{50}$ value for diosgenin against hCES2A was evaluated as low as $0.61 \mu \mathrm{M}$. In addition, loperamide (LPA), a known hCES2A inhibitor, was tested under the same condition as positive control. The result indicated that LPA showed inferior inhibitory effect on hCES2A in contrast to diosgenin. The dose-dependent inhibition curves of diosgenin, polyphyllin VI, polyphyllin VII and LPA against hCES2A mediated FD hydrolysis are shown in figure 2 . These findings demonstrated that diosgenin could strongly inhibit the enzymatic activity of hCES2A.

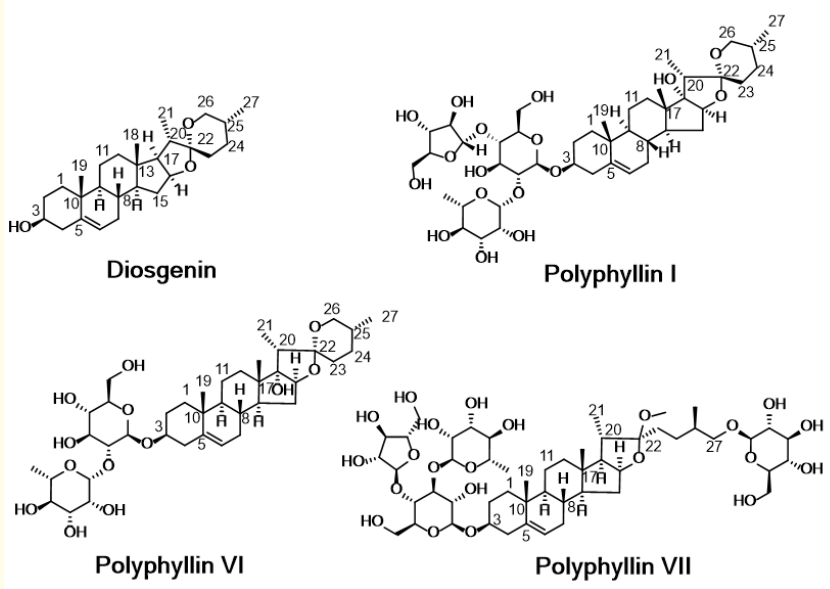

Figure 1: The structure of the four active ingredients of Paris polyphylla

\begin{tabular}{|c|c|c|c|c|}
\hline $\begin{array}{c}\text { Enzyme } \\
\text { source }\end{array}$ & $\begin{array}{c}\text { Target } \\
\text { enzyme }\end{array}$ & Substrate & $\begin{array}{c}\text { Active } \\
\text { ingredients }\end{array}$ & IC $_{\mathbf{5 0}}(\boldsymbol{\mu M})$ \\
\hline HLM & hCES2A & FD & Polyphyllin I & $>100$ \\
\hline HLM & hCES2A & FD & Polyphyllin VI & $11.95 \pm 2.78$ \\
\hline HLM & hCES2A & FD & Polyphyllin VII & $7.86 \pm 6.52$ \\
\hline HLM & hCES2A & FD & Diosgenin & $0.61 \pm 0.09$ \\
\hline HLM & hCES2A & FD & LPA & $1.75 \pm 0.64$ \\
\hline
\end{tabular}

Table 1: $\mathrm{IC}_{50}$ values of polyphyllin I, VI, VII and diosgenin toward hCES2A.

${ }^{a}$ All experimental data are averages of at least three independent experiments.

bLPA: Loperamide, a positive inhibitor against carboxylesterases. 
(A)

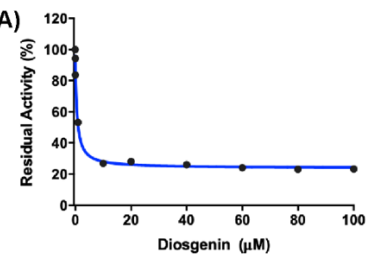

(C)

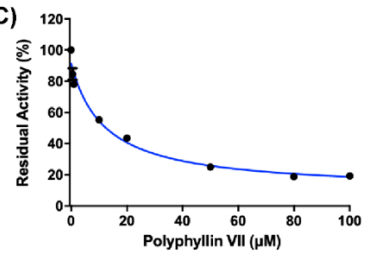

(B) 120

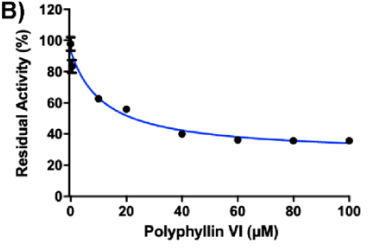

(D) 120

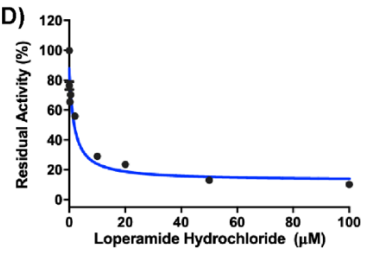

Figure 2: Diosgenin (A), Polyphyllin VI (B), Polyphyllin VII (C) and LPA (D) dose-dependently inhibited human hCES2A activity.

\section{Primary SAR study}

Polyphyllin I, VI, VII, and diosgenin were assayed for their inhibitory effects against hCES2A. As shown in figure 1, Polyphyllin I, VI, VII, and diosgenin have the similar steroid skeleton structure, but quite remarkably inhibitory effect on hCES2A. Polyphyllin I bearing a bulky hydrophilic group (1 furanose and 2 pyranose) at C3 showed poor inhibitory effect on hCES2A compared with diosgenin, while polyphyllin VI replaced the C3 group of polyphyllin I with a smaller group (1 furanose and 1 pyranose) led to an increase of the inhibitory effects on hCES2A. In addition, polyphyllin VII bearing hydrophilic group at C3 (1 furanose and 2 pyranose) and C27 (1 pyranose) displayed good inhibitory effect on hCES2A compared with polyphyllin I. It is evident from table 1 that diosgenin has a better inhibitory effect on hCES2A over polyphyllin I, polyphyllin VI and polyphyllin VII. This may be resulted from that diosgenin is relatively easy to enter the hydrophobic cavity sites of hCES2A, and the large hydrophilic groups at C3 and C27 of polyphyllin I, VI, VII make them more difficult to enter the relatively narrow cavity sites of hCES2A.

Inhibition mechanism analyses of diosgenin towards hCES2A

The potent inhibition potency of diosgenin against hCES2A encouraged us further to investigate the inhibition mechanism of diosgenin toward hCES2A. As shown in figure 3, the inhibitory be-

havior of diosgenin against hCES2A-mediated FD hydrolysis was performed in the HLMs. The results demonstrated that diosgenin potently inhibited hCES2A-mediated FD hydrolysis in a mixed inhibition manner in HLM, with the $K_{\mathrm{i}}$ values of $1.15 \mu \mathrm{M}$.
(A)

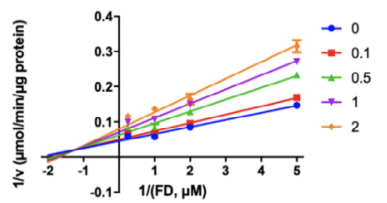

(B)

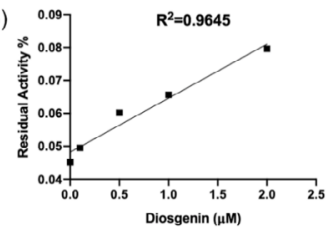

Figure 3: A) The Lineweave-Burk plots of Diosgenin against hCES2A-catalyzed FD hydrolysis. B) The second plot of slopes from A.

Selectivity of diosgenin towards hCES2A over other human serine hydrolases

To test whether diosgenin has similar inhibitory effects on other serine hydrolases, we evaluate the inhibitory effects of diosgenin on the specific substrates of hCES1A, PL, BChE, DPP-IV and Thrombin. The bioassay results are generalized in table 2. Diosgenin exhibited extremely potent inhibitory effect on hCES2A, with the $\mathrm{IC}_{50}$ value of $0.61 \mu \mathrm{M}$, and possessed significantly higher selectivity than hCES1A. According to the known assays [1,13], human serine hydrolases including PL, BChE, DPP-IV and thrombin were carried out to prove the selectivity of diosgenin on hCES2A. Diosgenin also exhibited extremely low inhibitory effect on Lipase ( IC $\left._{50}, 4.22 \mu \mathrm{M}\right)$. In addition, BChE, DPP-IV, and Thrombin, were hardly inhibited by diosgenin $\left(\mathrm{IC}_{50}\right.$ values $\left.>100 \mu \mathrm{M}\right)$.

\begin{tabular}{|c|c|c|c|}
\hline Enzyme source & Target enzyme & Substrate & IC $_{\mathbf{5 0}}(\boldsymbol{\mu M})$ \\
\hline HLM & hCES2A & FD & $0.61 \pm 0.09$ \\
\hline $\begin{array}{c}\text { Porcine pancre- } \\
\text { atic lipase }\end{array}$ & hCES1A & DME & $>100$ \\
\hline Human plasma & BChE & 4-MUO & $4.22 \pm 0.29$ \\
\hline Human plasma & DPP-IV & GP-BAN & $>100$ \\
\hline Thrombin & Thrombin & $\begin{array}{c}\text { Z-Gly-Gly- } \\
\text { Arg-AMC }\end{array}$ & $>100$ \\
\hline
\end{tabular}

Table 2: $\mathrm{IC}_{50}$ values of diosgenin toward hCES2A, hCES1A, PL, DPP-IV, BChE and thrombin. 
Molecular docking simulations

To further investigate the molecular mechanism of interactions between hCES2A and diosgenin, we perform molecular docking. As depicted in figure 4 , diosgenin could be well-docked into the active site and z-site, with the binding energy of $-8.7 \mathrm{kcal} / \mathrm{mol}$ for each site, suggesting that hydrophobic interactions might be the major forces for the binding of diosgenin on this enzyme. These discoveries were highly consistent with the mix-type inhibitory manner of diosgenin. Besides, diosgenin was in contact with multiple residues by van der Waals interactions, see Figure 5 in supplementary Materials for details.

(A)

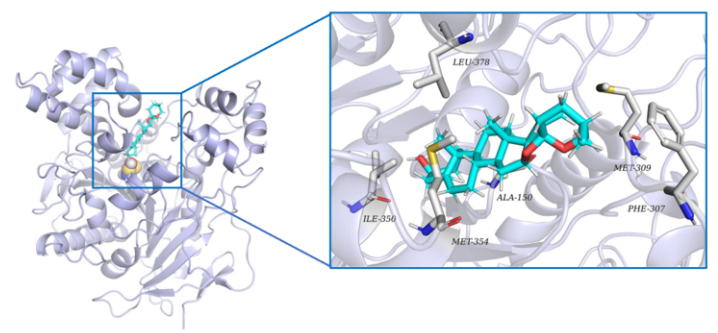

(B)

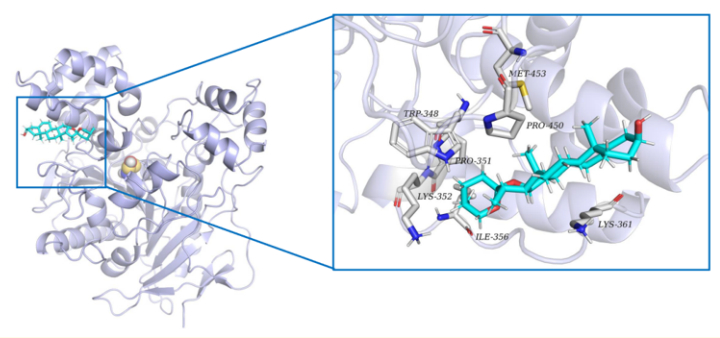

Figure 4: A) Diosgenin could be docked into the active catalytic site cavity of hCES2A, 2D interactions between diosgenin and the amino acid residues surrounding the active catalytic site of hCES2A. B) Diosgenin could be docked into the z-site of hCES2A, $2 \mathrm{D}$ interactions between diosgenin and the amino acid residues surrounding to the z-site of hCESA2.

\section{Cytotoxicity test}

Caco-2 cells were exposed to several concentrations of disgenin and polyphyllins for $48 \mathrm{~h}$ to establish a cellular model of enterotoxicity. Diogenin, polyphyllin I, polyphyllin VI, and polyphyllin VII produced concentration-dependent cytotoxicity in Caco- 2 cells, as determined by the CCK8 assay. As shown in Figure 6, diogenin exhibited relatively poor cytotoxicity to the human epithelial cell line
(A)

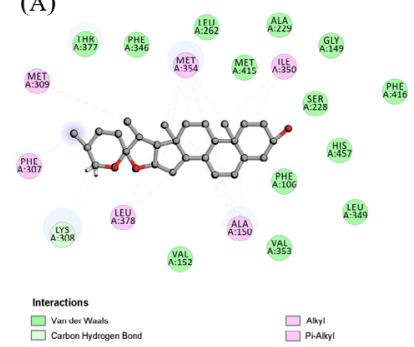

(B)

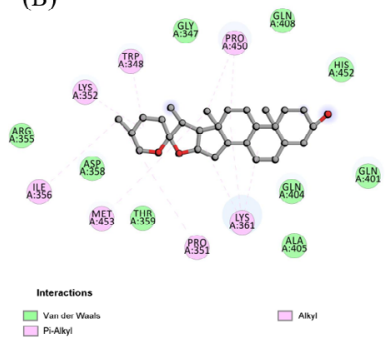

Figure 5: Diosgenin was in contact with multiple residues by van der Waals interactions.

Caco-2, the cell viability of the cell lines was more than $85 \%$ upon addition of diogenin with $10 \mu \mathrm{M}$ at $37^{\circ} \mathrm{C}$ for $48 \mathrm{~h}$. The median lethal dose $\left(\mathrm{LD}_{50}\right)$ of diosgenin was $34 \mu \mathrm{M}$, while polyphyllin I, polyphyllin VI and polyphyllin VII exhibited relatively good cytotoxicity with $\mathrm{LD}_{50}$ of $2.38 \mu \mathrm{M}, 4.20 \mu \mathrm{M}$ and $4.06 \mu \mathrm{M}$, respectively.
(A)

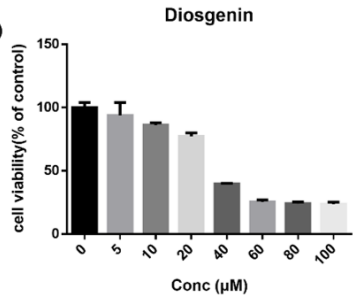

(C)

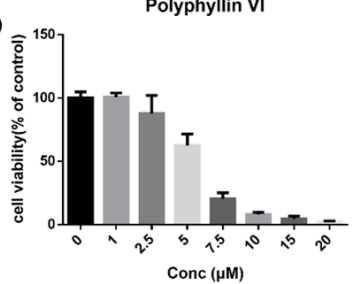

(B)

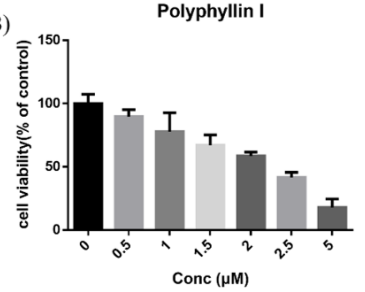

(D)

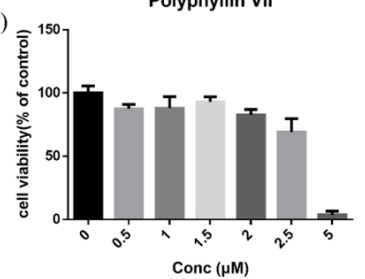

Figure 6: Diosgenin could be docked into the active catalytic site cavity of hCES2A, 2D interactions between diosgenin and the amino acid residues surrounding the active catalytic site of hCES2A. B) Diosgenin could be docked into the z-site of hCES2A, $2 \mathrm{D}$ interactions between diosgenin and the amino acid residues surrounding to the z-site of hCESA2. 


\section{Discussion and Conclusion}

hCES2A plays an important and irreplaceable role in the metabolic activation of many prodrugs, including anticancer drugs such as capecitabine and CPT-11, and this metabolic activation will also cause metabolic toxicity or decrease the efficacy to some extent. To improve the situation, it is necessary to use hCES2A inhibitors in combination $[9,31,39]$. More and more clinically important therapeutic drugs or endogenous chemicals and environmental toxicants are proven to be substrates of hCES2A, which emphasizes the importance of this enzyme in the human [27]. Therefore, the development and application of potential efficacious and highly selective hCES2A regulators are extremely important.

Traditional Chinese medicine (TCM) has been widely used in China's long history, especially in the prevention and treatment of various infectious diseases. In this COVID-19 epidemic, relying on its advantages in syndrome differentiation and treatment and overall curative effect, TCM has played a great role in reducing complications and reducing mortality [16].

With the continuous advancement of modern analysis and testing methods, the ingredients and effective ingredients of Chinese medicine are gradually clarified, the utilization rate of Chinese medicine is steadily increasing worldwide, and there are many cases of combining western medicine and traditional Chinese medicine to treat diseases $[30,36]$. Paris polyphylla is extensively used in traditional systems Chinese medicines mainly for its anticancerous property, research found that polyphyllin VII suppressed Colorectal cancer cell proliferation and migration, polyphyllin?and polyphyllin VI may exert a broad spectrum of anti-tumor effects [22,2425]. And diosgenin, one of its components, has been found to have antioxidant, anti-inflammatory and anti-cancer activities [4].

The inhibitory effects of diogenin towards hCES2A was measured using FD as the probe substrate and HLMs as the enzyme source. As shown in figure 2, the diosgenin significantly inhibited hCES2A in a concentration-dependent manner with $\mathrm{IC}_{50}$ is as low as $0.40 \mu \mathrm{M}$, however, it is worth noting that loperamide hydrochloride, a classical inhibitor of hCES2A, has a $K_{\mathrm{i}}$ value of 1.5 when 4-methyl-umbelliferone is used as the substrate [15]. In order to compare whether diosgenin has high selectivity to hCES2A, we studied the inhibitory effect of diosgenin on other enzymes of the same family. DME, GP-BAN, BTch, 4-MUO, Z-Gly-Gly-Arg-AMC was employed as specific probe substrate of hCES1A, DPP-IV, BChE, PL and Thrombin respectively to evaluate the inhibitory effect of diosgenin on them. The results showed that diosgenin had almost no inhibitory effect on hCES1A, DPP-IV, BChE and thrombin IC $_{50}$ $>100 \mu \mathrm{M}$ ), for PL, its inhibitory effect is strong, and its $\mathrm{IC}_{50}$ is 4.22 $\mu \mathrm{M}$, but its mechanism of action still needs further study. Those results showed that diosgenin had high selectivity for hCES2A and the inhibition type was mixed inhibition with the $K_{\mathrm{i}}$ value was 1.51 $\mu \mathrm{M}$. Molecular docking was a method to study the interaction between small molecular and protein, therefore, the potential interaction mechanism of diosgenin with human hCES2A was analyzed by molecular docking. The analysis results was shown in figure 4 diosgenin binds to the $\mathrm{Z}$ site, thereby inhibiting the hydrolysis of FD by hCES2A, and ultimately inhibiting the activity of hCES2A. From the diagram of $2 \mathrm{D}$ interactions between diosgenin and the amino acid residues surrounding to the z-site of hCES2A, diosgenin was in contact with multiple amino acid residues TRP-348, LYS-352, PRO351, ILE-356, MET-453, PRO-450, LYS-361 at the z-site by van der Waals interactions. The lowest binding energy of diosgenin with hCES2A was $-8.7 \mathrm{kcal} / \mathrm{mol}$. All the above mentioned results further confirmed the conclusion that diosgenin possessed potent hCES2A inhibitory activity, which indicated that diosgenin may be developed as a new highly effective inhibitor of hCES2A.

In summary, the inhibitory effects of the diosgenin on the catalytic activity of hCES2A were investigated for the first time, by using florescence-based enzyme inhibition assays. Finally, the diosgenin was found to have a strong inhibitory effect on hCES2A, and inhibition kinetic analyses demonstrated that diosgenin potently inhibited hCES2A in a mixed inhibition manner, with a $K_{\mathrm{i}}$ value of $1.51 \mu \mathrm{M}$. Above results will help to develop diosgenin into a more effective and selective hCES2A inhibitor, and and provide a theoretical basis for its potential biomedical applications.

\section{Notes}

The authors declare no competing financial interest.

\section{Acknowledgements}

This work was supported by the National Key Research and Development Program of China (2017YFC1702000), the National Science and Technology Major Project of China (2018ZX09731016004), the NSF of China (81703604, 81803489 and 81773687), and Clinical Advantage Discipline of Health System of Putuo District in Shanghai (2019ysxk01). 


\section{Conflicts of Interest}

The authors declare no conflict of interest.

\section{Highlights}

- The extract of diosgenin strongly inhibited Human carboxylesterase 2 (hCES2A) in a dose-dependent manner.

- The structure-activity relationship and inhibition mechanism of diosgenin and hCES2A were elucidated.

- It reflects the advantages of synergism and detoxification after the combination of Chinese and western medicine and conforms to the development direction of traditional Chinese medicine.

\section{Bibliography}

1. Bachovchin D A and B F Cravatt. "The Pharmacological Landscape and Therapeutic Potential of Serine Hydrolases". Nature Reviews Drug Discovery 11.1 (2012): 52-68.

2. Bencharit, S., et al. "Structural Basis of Heroin and Cocaine Metabolism by a Promiscuous Human Drug-Processing Enzyme". Nature Structural and Molecular Biology 10.5 (2003): 349-356.

3. Feng F F., et al. "Inhibitory Effects of Polyphyllins I and Vii on Human Cisplatin-Resistant Nsclc Via P53 Upregulation and Cip2a/Akt/Mtor Signaling Axis Inhibition". Chinese Journal of Natural Medicines 17.10 (2019): 768-777.

4. Gupta D D., et al. "Evaluation of Antioxidant, Anti-Inflammatory and Anticancer Activities of Diosgenin Enriched Paris Polyphylla Rhizome Extract of Indian Himalayan Landraces". Journal of Ethnopharmacology 270 (2021): 113842.

5. Hatfield M J., et al. "Modulation of Esterified Drug Metabolism by Tanshinones from Salvia Miltiorrhiza ("Danshen”)". Journal of Natural Products 76.1 (2013): 36-44.

6. Hosokawa, M. "Structure and Catalytic Properties of Carboxylesterase Isozymes Involved in Metabolic Activation of Prodrugs". Molecules 13.2 (2008): 412-431.

7. Hu Q., et al. "Inhibition of Pancreatic Lipase by Environmental Xenoestrogens". Ecotoxicology and Environmental Safety 192 (2020): 110305.
8. Huo P C., et al. "Design, Synthesis and Biological Evaluation of Indanone-Chalcone Hybrids as Potent and Selective Hces2a Inhibitors". European Journal of Medicinal Chemistry 209 (2021): 112856.

9. Lam S W., et al. "The Role of Pharmacogenetics in Capecitabine Efficacy and Toxicity". Cancer Treatment Reviews 50 (2016): 9-22.

10. Lee M S., et al. "Effects of Polyphyllin D, a Steroidal Saponin in Paris Polyphylla, in Growth Inhibition of Human Breast Cancer Cells and in Xenograft". Cancer Biology and Therapy 4.11 (2005): 1248-1254.

11. Li Y G., et al. "Fructus Psoraleae Contains Natural Compounds with Potent Inhibitory Effects Towards Human Carboxylesterase 2". Fitoterapia 101 (2015): 99-106.

12. Liu Y J., et al. "Identification and Characterization of Naturally Occurring Inhibitors against Human Carboxylesterase 2 in White Mulberry Root-Bark". Fitoterapia 115 (2016): 57-63.

13. Long J Z and B F Cravatt. "The Metabolic Serine Hydrolases and Their Functions in Mammalian Physiology and Disease". Chemical Review 111.10 (2011): 6022-6063.

14. Oh J., et al. "The Novel Carboxylesterase 1 Variant C.662a $>\mathrm{G}$ May Decrease the Bioactivation of Oseltamivir in Humans". PLoS One 12.4 (2017): e0176320.

15. Quinney SK., et al. "Hydrolysis of Capecitabine to 5'-Deoxy5-Fluorocytidine by Human Carboxylesterases and Inhibition by Loperamide". Journal of Pharmacology and Experimental Therapeutics 313.3 (2005): 1011-1016.

16. Ren JL., et al. "Traditional Chinese Medicine for Covid-19 Treatment". Pharmacology Research 155 (2020): 104743.

17. Ribelles, N., et al. "A Carboxylesterase 2 Gene Polymorphism as Predictor of Capecitabine on Response and Time to Progression". Current Drug Metabolism 9.4 (2008): 336-343.

18. Sanghani S P., et al. "Human Carboxylesterases: An Update on Ces1, Ces2 and Ces3". Protein and Peptide Letters 16.10 (2009): 1207-1214.

19. Sethi, G., et al. "Pro-Apoptotic and Anti-Cancer Properties of Diosgenin: A Comprehensive and Critical Review". Nutrients 10.5 (2018). 
20. Shaojun, C., et al. "Expression of Topoisomerase 1 and Carboxylesterase 2 Correlates with Irinotecan Treatment Response in Metastatic Colorectal Cancer". Cancer Biology and Therapy 19.3 (2018): 153-159.

21. Smith N F., et al. "Pharmacogenetics of Irinotecan Metabolism and Transport: An Update". Toxicology In Vitro 20.2 (2006): 163-175.

22. Song C., et al. "Polyphyllin Vii Suppresses Cell Proliferation, the Cell Cycle and Cell Migration in Colorectal Cancer". Oncology Letter 21.1 (2021): 25.

23. Song SS., et al. "Flavonoids as Human Carboxylesterase 2 Inhibitors: Inhibition Potentials and Molecular Docking Simulations". International Journal of Biological Macromolecules 131 (2019): 201-208.

24. Teng J F., et al. "Polyphyllin Vi Induces Caspase-1-Mediated Pyroptosis Via the Induction of Ros/Nf-Kb/Nlrp3/Gsdmd Signal Axis in Non-Small Cell Lung Cancer". Cancers (Basel) 12.1 (2020).

25. Tian Y., et al. "Anti-Cancer Effects of Polyphyllin I: An Update in 5 Years". Chemico-Biological Interactions 316 (2020): 108936.

26. Trott $O$ and A J Olson. "Autodock Vina: Improving the Speed and Accuracy of Docking with a New Scoring Function, Efficient Optimization, and Multithreading". Journal of Computational Chemistry 31.2 (2010): 455-461.

27. Wang DD., et al. "Recent Progress in the Discovery of Natural Inhibitors against Human Carboxylesterases”. Fitoterapia 117 (2017): 84-95.

28. Wang D., et al. "Human Carboxylesterases: A Comprehensive Review". Acta Pharmaceutica Sinica B 8.5 (2018): 699-712.

29. Wang Y Q., et al. "Nevadensin Is a Naturally Occurring Selective Inhibitor of Human Carboxylesterase 1". International Journal of Biological Macromolecules 120.Pt B (2018): 1944-1954.

30. Wong C C., et al. "Carboxylesterases 1 and 2 Hydrolyze Phospho-Nonsteroidal Anti-Inflammatory Drugs: Relevance to Their Pharmacological Activity". Journal of Pharmacology and Experimental Therapeutics 340.2 (2012): 422-432.
31. Xing Q., et al. "Efficacy and Safety of Integrated Traditional Chinese Medicine and Western Medicine on the Treatment of Rheumatoid Arthritis: A Meta-Analysis". Evidence-Based Complementary and Alternative Medicine 2020 (2020): 4348709.

32. Xu J., et al. "Potential Pharmacokinetic Herb-Drug Interactions: Have We Overlooked the Importance of Human Carboxylesterases 1 and 2?" Current Drug Metabolism 20.2 (2019): 130-137.

33. Yang, D., et al. "Human Carboxylesterases Hce1 and Hce2: Ontogenic Expression, Inter-Individual Variability and Differential Hydrolysis of Oseltamivir, Aspirin, Deltamethrin and Permethrin". Biochemistry and Pharmacology 77.2 (2009): 238-247.

34. Yano H., et al. "Overexpression of Carboxylesterase-2 Results in Enhanced Efficacy of Topoisomerase I Inhibitor, Irinotecan (Cpt-11), for Multiple Myeloma". Cancer Science 99.11 (2008): 2309-2314.

35. Zhang H F., et al. "Inhibitory Effects of Polyphyllin Vii on the Migration and Invasion of Colon Cancer Cells and the Mechanism". Immunological Journal 034.004 (2018): 286-293.

36. Zhang J., et al. "A High-Throughput Screening Assay for Dipeptidyl Peptidase-Iv Inhibitors Using Human Plasma". Annals of Methods (2021).

37. Zhang, J., et al. "A Review of Behcet's Disease from the Perspectives of Both Western and Chinese Medicine". Journal of Traditional Chinese Medical Sciences 39.1 (2019): 139-152.

38. Zhao Y S., et al. "Discovery of Natural Alkaloids as Potent and Selective Inhibitors against Human Carboxylesterase 2". Bioorganic Chemistry 105 (2020): 104367.

39. Zou L W., et al. "Carboxylesterase Inhibitors: An Update”. Current Medicinal Chemistry 25.14 (2018): 1627-1649.

40. Zou L W., et al. "Design, Synthesis, and Structure-Activity Relationship Study of Glycyrrhetinic Acid Derivatives as Potent and Selective Inhibitors against Human Carboxylesterase 2". European Journal of Medicinal Chemistry 112 (2016): 280288. 
Discovery of Diosgenin as Potent and Selective Inhibitors Against Human Carboxylesterase 2

41. Zou L W., et al. "A Highly Specific Ratiometric Two-Photon Fluorescent Probe to Detect Dipeptidyl Peptidase Iv in Plasma and Living Systems". Biosensors and Bioelectronics 90 (2017): 283-289.

Volume 5 Issue 12 December 2021

(C) All rights are reserved by Xiao-Dong Li., et al. 